this was thought to be mostly a problem in the United States, where the lifetime rate of prescription drug misuse, for those aged 12 years or more, is as high as $20 \%$. However, a recent study shows that the European Union is not far behind, with lifetime rates between $7 \%$ and $13 \%$ for substances such as opioid analgesics. The European Union Medicine Study collected the data from a population of 20000 adults and 2000 teenagers. Spain, Sweden and the UK have high rates of misuse, while Germany has the lowest rates. Interestingly, the internet was the least common way of getting hold of prescription drugs.

Among those misusing prescription drugs there were high rates of poly-drug use: $52 \%$ of those prescribed stimulants, $32 \%$ of prescribed opioid users and $28 \%$ of those on prescribed sedatives also used illicit drugs.

This is the first comparative study of prescription drug misuse in the European Union and it raises an alarm about a problem that may increase if no appropriate measures are taken.

Novak, S. P., Håkansson, A., Martinez-Raga, J., et al (2016) Nonmedical use of prescription drugs in the European Union. BMC Psychiatry. doi: 10.1186/s12888-016-0909-3.

\section{Is life precious enough for all?}

World Suicide Prevention Day was 10 SepVtember 2016. It was organised by the International Association for Suicide Prevention (IASP) and the World Health Organization (WHO). This serves as a reminder for all those involved with people at suicide risk to up their act.

According to the WHO, every 40 seconds one person somewhere in the globe puts an end to his or her life: a total of 800000 people every year. Globally, suicide rates have been increasing.

The 25 countries with the highest suicide rates differ significantly from each other in a variety of ways, including geographical position, culture and socioeconomic conditions. Almost all continents are represented and men outnumber women in all countries by a factor of 2-3 or more in many cases. The countries with the highest suicide rates are, in ascending order, Poland at 16.6 per 100000 (30.5 among men; 3.8 among women), Ukraine, Sudan, Comoros, Bhutan, Zimbabwe, Belarus, Japan, Hungary, Uganda, Russian Federation, South Sudan, Turkmenistan, Burundi, Kazakhstan, Nepal, Tanzania, Mozambique, Surinam, Lithuania, Sri Lanka, South Korea and North Korea. The South American country Guyana tops the list with a suicide rates of 44.2 per 100000 (70.8 among men; 22.1 among women). Perhaps the adequacy of the methodology in different countries may be not as high as would be desirable, and the resulting data may be questioned for accuracy, but the figures cannot be completely dismissed. There is a pattern that cannot be ignored.

Although suicide is in our domain when due to mental illness and there is no question that, as psychiatrists, we should be doing our utmost to reduce suicide rates, can we really make a big difference globally? Aren't conflict, poverty, oppression and dramatic political and economic changes that turn people's lives upside down at the root of many suicides, or, dare I say, mostly responsible for people opting for death? Shouldn't the politicians and primarily the world leaders be discussing such matters? Should it be on the G20 agenda?

World Health Organization. Suicide Rates Data By Country. WHO, 2012.

\section{Enjoy your coffee - it is good for you!}

Tnlike other stimulants, coffee has never been found to be associated with any harm to human beings. A recent meta-analysis to assess the influence of coffee drinking on the risk of developing cognitive disorders is more than reassuring for us coffee addicts! Nine prospective cohort studies involving 34282 participants were included, with follow-up ranging from 1.3 to 28 years. Daily drinking of one or two cups of coffee was associated with a lower occurrence of cognitive disorders, including Alzheimer's disease.

Well you now know that coffee is good for your brain, but did you know that it is also good for your liver? Evidence shows that it reduces liver enzymes in viral hepatitis, non-alcoholic fatty liver disease (NAFLD) and cirrhosis, and it also offers some protection from hepatocellular carcinoma. However, how your coffee is made matters. It appears that filter coffee may be best for you, rather than espresso and barista. It filters out some bad-for-you substances such as kahweol and cafestol, which are caffeine diterpenes, released from ground coffee beans but removed by the paper filters.

The bad news for tea drinkers and those favouring other caffeine drinks is that they don't have the benefits that coffee offers; it seems that substances in addition to caffeine, contained in coffee, are also needed.

Wu, L., Sun, D. \& He, Y. (2016) Coffee intake and the incident risk of cognitive disorders: a dose-response meta-analysis of nine prospective cohort studies. Clinical Nutrition [Epub ahead of print]. doi: 10.1016/j.clnu.2016.05.015. PMID: 27288328

Saab, S., Mallam, D., Cox, II, J. A., et al (2014) Impact of coffee on liver diseases. A systematic review. Liver International, 34 (4), 495-504.

\section{E-cigarettes - to use or not to use?}

$\mathrm{f}$, as you are enjoying your coffee, you get tempted to light a cigarette, remind yourself that, unlike coffee, cigarettes are proven to cause serious harm! What about e-cigarettes? There are concerns that e-cigarettes' increasing popularity may be undermining successful quitting and this would have an adverse effect on public health. A recent publication in the $B M J$ examined the prevalence of e-cigarette use in current smokers and during a quit attempt, to predict quit success. Don't throw your e-cigarette away! The authors found that changes in the prevalence of e-cigarette use in England have been positively associated with the success rates of quit attempts.

Beard, E., West, R., Michie, S., et al (2016) Association between electronic cigarette use and changes in quit attempts, success of quit attempts, use of smoking cessation pharmacotherapy, and use of stop smoking services in England: time series analysis of population trends. BMJ, 354, i4645. doi: http://dx.doi.org/10.1136/bmj.i4645. 